\title{
Role of striatum in the pause and burst generation in the globus pallidus of 6-OHDA-treated rats
}

\author{
Hitoshi Kita* and Takako Kita \\ Department of Anatomy and Neurobiology, College of Medicine, The University of Tennessee Health Science Center, Memphis, TN, USA
}

\section{Edited by:}

James M. Tepper, Rutgers, The State

University of New Jersey, USA

Reviewed by:

Alessandro Stefani, University of

Rome, Italy

Judith Walters, National Institute of

Neurological Disease and Stroke,

National Institutes of Medicine, USA

*Correspondence:

Hitoshi Kita, Department of Anatomy and Neurobiology, College of Medicine,

The University of Tennessee Health

Science Center, 855 Monroe Avenue,

Memphis, TN 38163, USA.

e-mail:hkita@uthsc.edu
Electrophysiological studies in patients and animal models of Parkinson's disease (PD) often reported increased burst activity of neurons in the basal ganglia. Neurons in the globus pallidus external (GPe) segment in 6-hydroxydopamine (6-OHDA)-treated hemi-parkinsonian rats fire with strong bursts interrupted by pauses. The goal of this study was to evaluate the hypothesis that dopamine (DA)-depletion increases burst firings of striatal (Str) neurons projecting to the GPe and that the increased Str-GPe burst inputs play a significant role in the generation of pauses and bursts in GPe and its projection sites. To evaluate this hypothesis, the unitary activity of Str and GPe was recorded from control and 6-OHDA-treated rats anesthetized with 0.5-1\% isoflurane. The occurrence of pauses and bursts in the firings of GPe neurons was significantly higher in 6-OHDA than in normal rats. Muscimol injection into the Str of 6-OHDA rats increased average firing rate and greatly reduced the pauses and bursts in GPe. Recordings from Str revealed that most of the presumed projection neurons in control rats have very low spontaneous activity, and even the occasional neurons that did exhibit spontaneous burst firings did so with an average rate of less than $2 \mathrm{~Hz}$. In DA-depleted Str, neurons having stronger bursts and a higher average firing rate were encountered more frequently. Juxtacellular labeling revealed that most of these neurons were medium spiny neurons projecting only to GPe. Injection of a behaviorally effective dose of methyl-L-DOPA into the Str of 6-OHDA rats significantly increased the average firing rate and decreased the number of pauses of GPe neurons. These data validate the hypothesis that DA-depletion increases burst firings of Str neurons projecting to the GPe and that the increased Str-GPe burst inputs play a significant role in the generation of pauses and bursts in $\mathrm{GPe}$. These results suggest that treatment to reduce burst Str-GPe inhibitory inputs may help to restore some PD disabilities.

Keywords: dopamine depletion, pauses, burst, firing pattern, unitary activity, globus pallidus, striatum, basal ganglia

\section{INTRODUCTION}

Electrophysiological observations made in patients and animal models of basal ganglia diseases reported various changes in the activity of basal ganglia neurons including abnormal firing patterns, increased bursts and pauses, and abnormal synchronized oscillations (Pan and Walters, 1988; Filion and Tremblay, 1991; Bergman et al., 1994; Ni et al., 2000; Soares et al., 2004; Wichmann and DeLong, 2006; Stefani et al., 2011). Among these, the most often observed changes are increased burst and pause activity of neurons in the basal ganglia, particularly in the pallidum and the subthalamic nucleus (STN; Pan and Walters, 1988; Filion and Tremblay, 1991; Bergman et al., 1994; Ni et al., 2000; Soares et al., 2004; Wichmann and DeLong, 2006). The increased bursts and pauses alone may not directly lead to the manifestation of basal ganglia diseases, but they do interfere with the processing of information required to execute coordinated movements and thus may indirectly influence the development of various basal ganglia diseases.

Because DA-depletion has been shown to increase the firing activity of striatal-globus pallidus external (Str-GPe) projection neurons by changing their synaptic activity and membrane properties (Hernandez-Lopez et al., 2000; West and Grace, 2002;
Mallet et al., 2006; Shen et al., 2008; Flores-Barrera et al., 2010; Surmeier et al., 2010) and because in vivo studies have shown that the firing activity of Str projection neurons greatly increases after DA-depletion (Pang et al., 2001; Tseng et al., 2001; West and Grace, 2002; Mallet et al., 2006), it seems possible that increased Str activity plays substantial roles in the generation of abnormal firings in the basal ganglia (Walters et al., 2007; Walters and Bergstrom, 2010). Thus, our hypothesis is that DA-depletion increases burst firings of Str neurons projecting to the GPe and that these increased Str burst inputs contribute significantly to the generation of pauses and bursts in GPe neurons. DA-depletion may also increase the irregular activity of GPe neurons by other mechanisms such as increasing synchronous activity in the cortico-STN-GPe projection and removing DA suppression of N-type calcium channels in GPe neurons (Stefani et al., 2002; Galati et al., 2009). We hypothesize that the strong, irregular burst, and pause activity of GPe contributes greatly to the generation of irregular activity in neurons of the basal ganglia output nuclei, the internal segment of the globus pallidus (GPi), and the substantia nigra pars reticulata $(\mathrm{SNr})$, which then disables information processing capability at their projection sites in the thalamus and brainstem (Stefani et al., 2011). 
To test this hypothesis, unit recordings of Str and GPe neurons were performed in isoflurane anesthetized control and 6-hydroxydopamine (6-OHDA)-treated rats (6-OHDA rats), the rodent model of Parkinson's disease (PD). The results indicate that DA-depletion increases burst firings of Str-GPe projection neurons, and the intra-Str injection of the GABA $_{A}$ agonist muscimol to block Str activity reduced the pauses and bursts in GPe firing. This report also provides evidence that intra-Str injection of a behaviorally effective dose of the DA precursor methyl-L-DOPA can decrease the pauses and the bursts. These results support the hypothesis that increased burst firings of Str-GPe projection neurons play substantial roles in the generation of abnormal firing patterns in the GPe and that this increased abnormal GPe activity may then induce abnormal activity in the entire basal ganglia and be a cause of psychomotor disabilities in PD. It can be further suggested that treatment to reduce burst Str-GPe inhibitory inputs may help to restore some of these disabilities.

\section{MATERIALS AND METHODS}

The experiments were performed on adult male Sprague Dawley rats (300-380 g) in compliance with the National Institutes of Health Guide for Care and Use of Laboratory Animals and the University of Tennessee Health Science Center Guide for the Use and Care of Laboratory Animals in Research.

\section{BEHAVIORAL TESTS}

All rats received 2 kinds of behavioral tests 2 times before and 2-4 times after the DA-depletion surgery described below. The rotational asymmetry test monitored the ambulatory movements of rats in a 1-m diameter chamber for 15 min using a video monitor system (Viewer, Biobserve, Konigswinter, Germany), which could identify and track the rats' head, body, and tail positions frame by frame (30 frames/s). The body location data were used to calculate the distance and direction of the body movement between frames. For the limb use asymmetry test, rats were placed in a $28-\mathrm{cm}$ diameter acrylic cylinder and videotaped for $10 \mathrm{~min}$. The number of times the left and right forelimbs touched the wall of the cylinder were counted.

\section{DA-DEPLETION AND HEAD HOLDER INSTALLATION}

After the behavioral tests, 10 rats were anesthetized with a mixture of Ketamine ( $85 \mathrm{mg} / \mathrm{kg}$, i.p.) and Xylazine (15 mg/kg, i.p.), treated with desmethylimipramine $(25 \mathrm{mg} / \mathrm{kg}$, i.p.) and pargyline $(5 \mathrm{mg} / \mathrm{kg}$, i.p.), and placed in a stereotaxic apparatus. A craniotomy was performed on the skull, and 6-OHDA ( $8 \mu \mathrm{g}$ in $4 \mu \mathrm{l}$ of saline containing $0.1 \%$ ascorbic acid) in six rats or the vehicle in four rats was injected into the left ascending mesotelencephalic DA-bundle. The 6-OHDA or vehicle was delivered slowly over a period of $10 \mathrm{~min}$ through a glass micropipette (tip diameter of about $50 \mu \mathrm{m}$ ) glued to the needle of a 10- $\mu$ l Hamilton syringe. The syringe plunger was advanced by a pulse motordriven actuator. After 2 weeks of recovery, the behavioral tests were performed again. Four of the six rats injected with 6-OHDA developed a strong left turn preference in the rotational asymmetry test and a strong left forelimb use preference $(<0.2 \mathrm{right} / \mathrm{left}$ touches in the cylinder test). The two 6-OHDA rats that failed to meet these behavioral criteria developed akinesia. These two rats were rejected for the unit recording study in order to constrain the experimental group to one set of behavioral changes. None of the four rats injected with the vehicle developed behavioral asymmetries.

After the behavioral tests, a second surgery was performed on the control and 6-OHDA rats. The rats were anesthetized with a mixture of Ketamine $(85 \mathrm{mg} / \mathrm{kg}$, i.p.) and Xylazine $(15 \mathrm{mg} / \mathrm{kg}$, i.p.) and mounted on a stereotaxic apparatus. To fix the rats' heads painlessly to a stereotaxic frame, a plastic head holder made with polycarbonate and stainless steel pipes was placed on the dorsal surface of the skull and secured with dental acrylic. Also, a craniotomy was performed over the lateral agranular cortex, which is the motor cortex (MC) of rodents, and a parallel bipolar stimulus electrode $(100 \mu \mathrm{m}$ insulated stainless steel wires with tip separation of $0.6-0.7 \mathrm{~mm}$ ) was implanted. For two control and one 6-OHDA rats, a pair of silver ball electrodes was also placed on the dura matter, one covering the right frontal agranular cortex and another covering the cerebellum to monitor the electrocorticogram. Another craniotomy was performed over the pallidum for recording electrode penetration. A thin layer of dental acrylic was used to cover the recording hole until the hole was needed for the recording sessions. After the surgery, the rats were monitored every hour until they recovered from anesthesia. If the rats showed any sign of pain, buprenorphine $(0.01-0.05 \mathrm{mg} / \mathrm{kg}, \mathrm{i} . \mathrm{p}$. was administered.

\section{UNIT RECORDINGS}

Unit recordings began 5-7 days after installation of the head holder and stimulus electrodes, during which some gain in body weight and normal behavior was observed in the rats. For the recordings, the rats were anesthetized with $2.5 \%$ isoflurane in oxygen and mounted on the stereotaxic device using the head holder. During the recording, the rats were maintained with $0.5-1.0 \%$ isoflurane that was adjusted to eliminate spontaneous whisker movements as well as any body movement to MC stimulation. Each rat was recorded 2-3 times a week for 3-4 weeks. After 4 weeks, the accurate placement of the recording electrode became difficult due to the progressive increase in the thickness of the dura matter, and therefore, the recordings were stopped.

Single-unit recordings of Str and GPe neurons were made with insulated tungsten or Elgiloy alloy microelectrodes with an AC resistance of 1-2.5 M $\Omega$. For Juxtacellular labeling of Str neurons, glass micropipettes filled with $1 \mathrm{M} \mathrm{NaCl}$ and $2 \%$ Neurobiotin were used, and recorded neurons were labeled by a method described elsewhere (Pinault, 1996). This report includes only the neurons that responded to MC stimulation (300 $\mu$ s duration single pulse, strength $80 \mu \mathrm{A}$ ), which ensures that the neurons were in the same functional territories of Str and GPe that receive projections from the stimulus site. Unitary activity was amplified, passed through a 0.7 to $2-\mathrm{KHz}$ band-pass filter (A-M Systems Model 1800, home modified), and converted into digital data by a homemade window discriminator. Digitized units and electrocorticograms were recorded using a computer. The Str and GPe neurons were identified by their firing patterns and by $\mathrm{x}$-ray images of the skull and the electrode. The locations were also identified histologically at the end of the experiments as described below. 


\section{ANALYSIS OF UNIT ACTIVITY}

Globus pallidus external neurons were recorded from four control and four 6-OHDA rats, and Str neurons were recorded from two rats from each group. The data obtained from the rats were pooled into control and 6-OHDA groups because the firing property obtained from anesthetized rats within each group were similar enough to combine. The existence of the refractory period in the single-unit recordings was tested by calculating an autocorrelogram of digitized spikes for $50 \mathrm{~s}$ of for GPe and $120 \mathrm{~s}$ for Str. The digitized data were also used calculate the rate and the burst index of firing of neurons. Bursts were detected by the "surprise" method of Legendy and Salcman (1985) using a surprise value $\geq 3$ and with the number of spikes $\geq 3$ defining a burst. These values were adopted from an extensive study of burst activity in monkey pallidum and STN (Wichmann and Soares, 2006). The proportion of spikes in bursts (\%) was chosen as the index because this method has been used frequently in other studies. To detect pauses in GPe firing, gaps in the spike trains were filled with phantom spikes with an inter-spike-interval equal to the mean inter-spike-interval of real spikes, and the Legendy and Salcman method with the same criteria used for burst detection were applied to the phantom spikes to identify pauses (Figure 1A). For the frequency component analysis, the spike trains were transformed using a moving average filter into a signal containing the density of the spikes versus time. The width of the moving average filter was $20 \mathrm{~ms}$ to ensure that the cut off of the filter was $50 \mathrm{~Hz}$. In the power histograms, the values express the fraction of the total power in the 0 to $50-\mathrm{Hz}$ band.

\section{INTRA-STRIATAL INJECTIONS}

The intra-Str injection of muscimol $(0.04 \mu \mathrm{g}$ in $0.2 \mu \mathrm{l}$ saline $)$ or methyl-L-DOPA ( $4 \mu \mathrm{g}$ in $0.2 \mu \mathrm{l}$ saline) was performed in a similar manner to the 6-OHDA injection described above. The injection site in Str was first determined by observing the MC-induced unit responses that were recorded through the injection micropipette, and then the drugs were injected slowly $(0.04 \mu \mathrm{l} / \mathrm{min})$ with an electric actuator on the microsyringe plunger. The drug injection was performed with at least a 3-day interval between injections. The dose of methyl-L-DOPA was determined by the ambulatory direction and distance tests on 2 of the 6-OHDA rats (Kita and Kita, 2011). The dose of muscimol was based on previous observations that $0.04 \mu \mathrm{g}$ of muscimol blocked $\mathrm{MC}$-induced excitation in an approximately $0.5 \mathrm{~mm}$ radius of the injection area (data not shown).

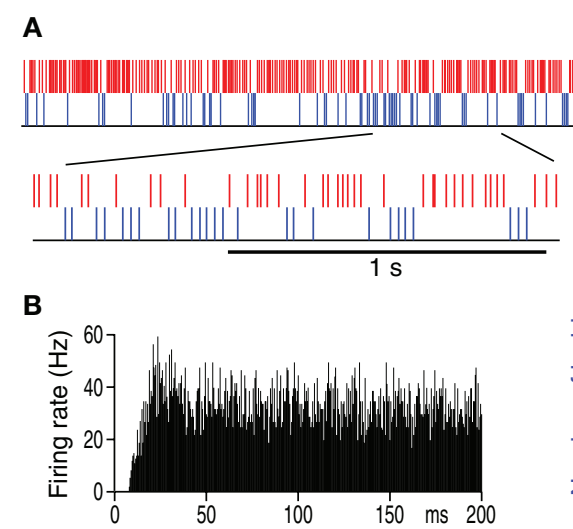

D

Average firing rate $=28.7 \mathrm{~Hz}$, burst index $=62.7 \%$

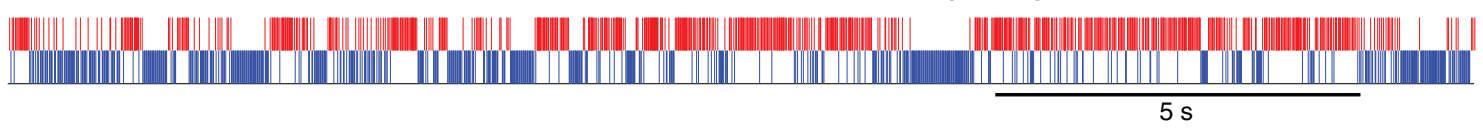

E

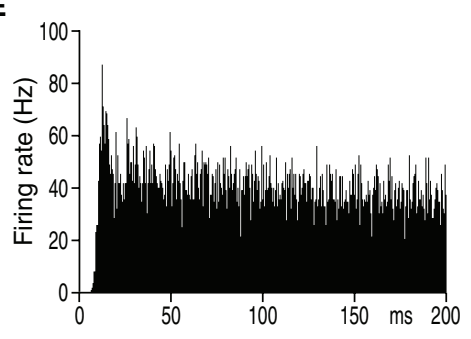

Average firing rate $=28.6 \mathrm{~Hz}$, burst index $=15.7 \%$

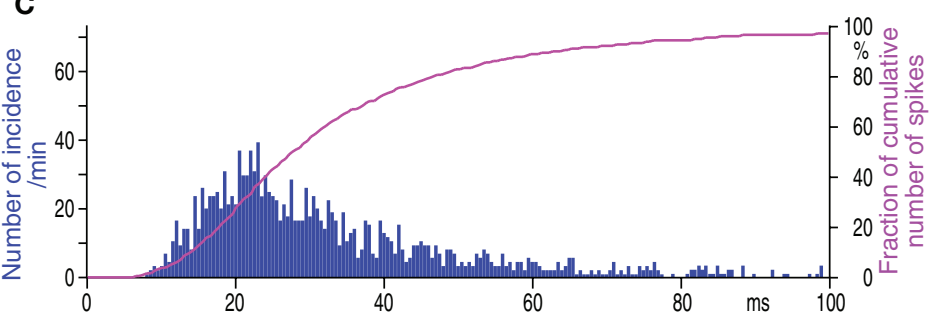

FIGURE 1 | Spontaneous firing patterns of a GPe neuron from a control (A-C) and from a 6-OHDA rat (D-F) having similar average firing rates. In $(\mathbf{A}, \mathbf{D})$, actual digitized spikes are shown in red. Blue phantom spikes occupying pauses have an inter-spike-interval equal to the mean inter-spike-intervals of

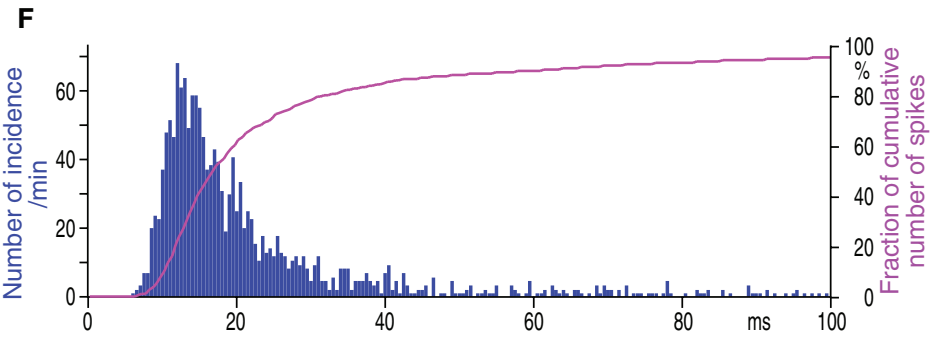

actual spikes. The phantom spikes were used for the detection of pauses (details in the section Materials and Methods). Autocorrelograms (B,E) and spike interval histograms $\mathbf{( C , F )}$ show that the neuron from the 6-OHDA rat has a larger peak at shorter inter-spike-intervals. 


\section{HISTOLOGY}

For the final experiment, $2-3$ recording sites were marked by passing a cathodal current $(10 \mu \mathrm{A}$ for $5 \mathrm{~s})$ through metal recording electrodes. About 1-2 days after the lesion, the rats were deeply anesthetized with a mixture of Ketamine $(100 \mathrm{mg} / \mathrm{Kg})$ and Xylazine (20 $\mathrm{mg} / \mathrm{Kg}$ ) and were perfused through the heart with 10-20 ml of isotonic saline followed by a fixative. The fixative was a mixture of $4 \%$ formaldehyde and $0.2 \%$ picric acid in a $0.12-\mathrm{M}$ sodium phosphate buffer (200-300 ml, pH 7.4). After perfusion, the brains were removed and postfixed overnight at $4^{\circ} \mathrm{C}$ and then equilibrated in a $10 \%$, followed by a $30 \%$, phosphate buffered ( $\mathrm{pH} 7.4$ ) sucrose solution. The brains were cut into $40 \mu \mathrm{m}$ sagittal sections on a freezing microtome. Sections from Neurobiotin injected brains were incubated for $5 \mathrm{~h}$ in phosphate buffered saline (PBS) containing $0.5 \%$ skim milk, $0.05 \%$ Triton X-100, and ABC Elite (1:150 dilution; Vector). After several washes with PBS, the sections were incubated for 10-20 min in $0.05 \mathrm{M}$ Tris-HCl buffered saline ( $\mathrm{pH} 7.6$ ) containing $0.04 \%$ diaminobenzidine tetrahydrochloride, $0.04 \% \mathrm{NiCl}_{2}$, and $0.001 \% \mathrm{H}_{2} \mathrm{O}_{2}$ to reveal the biotin containing neurons. Some of the sections were immunostained for tyrosine hydroxylase (TH), and others were stained with $1 \%$ cresyl violet to verify the recording sites. The sections for TH staining were incubated first for $5 \mathrm{~h}$ in PBS containing 0.5\% skim milk and $0.05 \%$ Triton X-100 and then for $48 \mathrm{~h}$ in PBS containing a monoclonal mouse anti-TH antibody (Sigma, 1:4,000), 0.5\% skim milk, and 0.05\% Triton X-100. Subsequently, the sections were incubated with biotinylated goat anti-mouse IgG antibody (1:200 dilution; Vector, Burlingame, CA, USA) in the PBS incubation medium for $2 \mathrm{~h}$. The sections were treated with $\mathrm{ABC}$ and diaminobenzidine tetrahydrochloride as described in the Neurobiotin sections. The sections were mounted on gelatin-coated slides, air-dried, dehydrated in graded alcohols to xylene, and coverslipped. All of the chemicals used for making the fixative, buffers, and other histological solutions were obtained from Sigma-Aldrich (St. Louis, MO, USA). Neurobiotin labeled axons were traced using a light microscope with a $\times 20$ or a $\times 40$ objective and a camera lucida (Olympus BX50).

\section{RESULTS \\ DA-DEPLETION INCREASES PAUSES AND BURSTS IN GPe FIRINGS}

Many previous observations of GPe neurons in patients or animal models of PD focused on the changes in the average firing rate and the generation of burst firings (Pan and Walters, 1988; Filion and Tremblay, 1991; Bergman et al., 1994; Ni et al., 2000; Soares et al., 2004; Wichmann and DeLong, 2006). Many of these observations were performed under local anesthesia or unanesthetized animals. The aims of the present analysis of GPe neurons were first, to evaluate whether or not increased burst firings of GPe neurons in 6-OHDA rats can be seen under isoflurane anesthesia, thus validating experimental conditions, and second, to evaluate whether any increased bursts were accompanied by an increased occurrence of pauses in the firings.

The spontaneous activity of 52 GPe neurons recorded from control and 54 from 6-OHDA rats was analyzed. The GPe neurons included in this report were of the high frequency firing with pause type, which are the most common type found in GPe (DeLong, 1971). The neurons with a slow, less than $10 \mathrm{~Hz}$, rhythmic firing of large, broad spikes were encountered mainly in the caudomedial and ventral regions of the nucleus and were excluded from the present analysis. The analysis of the spontaneous firing patterns revealed that the mean firing rate was similar in the control and 6 -OHDA rats, but the burst index was significantly higher in GPe of 6-OHDA rats (Table 1). This result indicates that the increase in burst activity can be seen in $0.5-1 \%$ isoflurane anesthetized 6-OHDA rats. The analysis also revealed that both the number of pauses and the fraction of total time occupied by the pauses were significantly higher in 6-OHDA rats (Table 1). Figure 1 shows an example of a control and a 6-OHDA GPe neuron with similar average firing rates. The neuron from a 6-OHDA rat has a higher burst index that was associated with an occurrence of a large number of longer pauses. The autocorrelograms and inter-spike-interval histograms show that the neuron from the 6-OHDA rat has a larger peak at shorter inter-spike-interval distributions compared with the neuron from the control rat.

\section{MUSCIMOL INJECTION INTO Str}

It can be assumed that if Str-GPe projections play a role in the generation of pauses and bursts in the firings of GPe neurons, silencing the activity of Str projection neurons by a local injection of the $\mathrm{GABA}_{\mathrm{A}}$ receptor agonist muscimol should decrease the pauses and bursts in GPe. In the experiment, MC-induced multi-unit excitatory responses were recorded using a micropipette containing muscimol to place the tip of the pipette to the middle of the MC-responsive area of the Str. Then, a neuron in the sensorimotor territory of GPe was recorded before and 10-20 min after muscimol injection $(0.04 \mu \mathrm{g}$ in $0.2 \mu \mathrm{l}$ saline, $0.04 \mu \mathrm{l} / \mathrm{min})$.

A total of 8 GPe neurons in 6-OHDA rats was tested by intra-Str injection of muscimol (Table 2). Muscimol significantly increased the average firing rate of GPe neurons, which indicated that Str provides substantial inhibition to GPe and that the muscimol did not spread into the GPe recording sites. Muscimol also significantly decreased the number of pauses, the fraction of time occupied by pauses, the burst index, and the $<3 \mathrm{~Hz}$ components of the firing frequency distribution of the firings in GPe neurons (Table 2; Figure 2). However, the autocorrelograms and inter-spike-interval distributions show that muscimol did not abolish the high frequency firing components having a peak at inter-spike-interval distributions $\approx 18 \mathrm{~ms}$. These results provide strong support for the hypothesis that Str-GPe inputs provide the driving force for the generation of pauses and bursts in GPe firings. The data also suggest that other excitatory driving forces such as STN glutamatergic inputs drive high frequency firings of GPe neurons.

Table 1 | Properties of spontaneous firings of of GPe neurons in control and 6-OHDA rats.

\begin{tabular}{llll}
\hline & Control, $\boldsymbol{n}=\mathbf{5 2}$ & & 6-OHDA, $\boldsymbol{n}=\mathbf{5 4}$ \\
\hline Firing rate & $29.3 \pm 12.2$ & $\mathrm{~ns}$ & $26.2 \pm 10.2$ \\
Burst index (\%) & $13.1 \pm 14.2$ & $p<0.0001$ & $44.3 \pm 15.6$ \\
Pause fraction (\%) & $11.9 \pm 4.1$ & $p<0.0001$ & $23.2 \pm 5.2$ \\
Number of pauses/min & $26.8 \pm 17.3$ & $p<0.0001$ & $55.6 \pm 14.8$ \\
\hline
\end{tabular}

Data: mean \pm SD; statistics: Mann-Whitney U-test; ns: not significant; pause fraction: fraction of total time occupied by pauses. 
Table 2 | Effects of intra-Str injections of methyl-L-DOPA and muscimol on firing properties of GPe neurons in 6-OHDA rats.

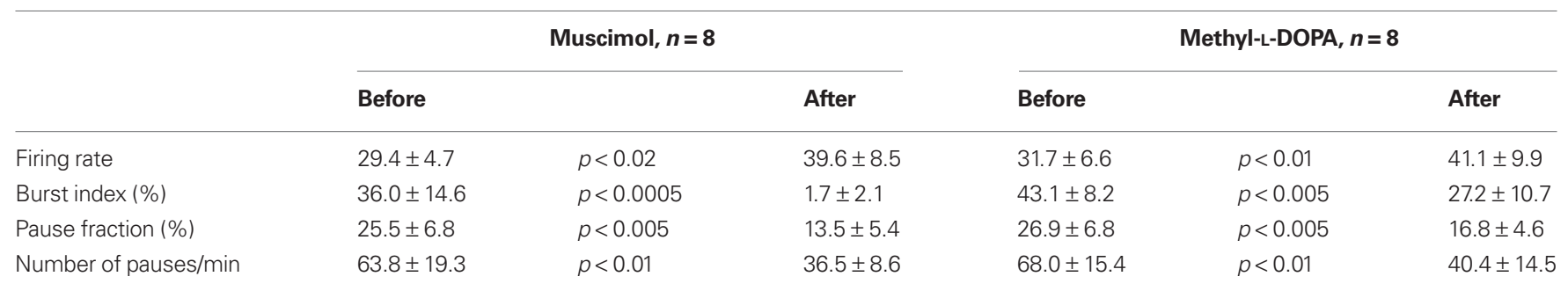

Data: mean \pm SD; statistics: paired t-test; pause fraction: fraction of total time occupied by pauses longer than 100 ms.

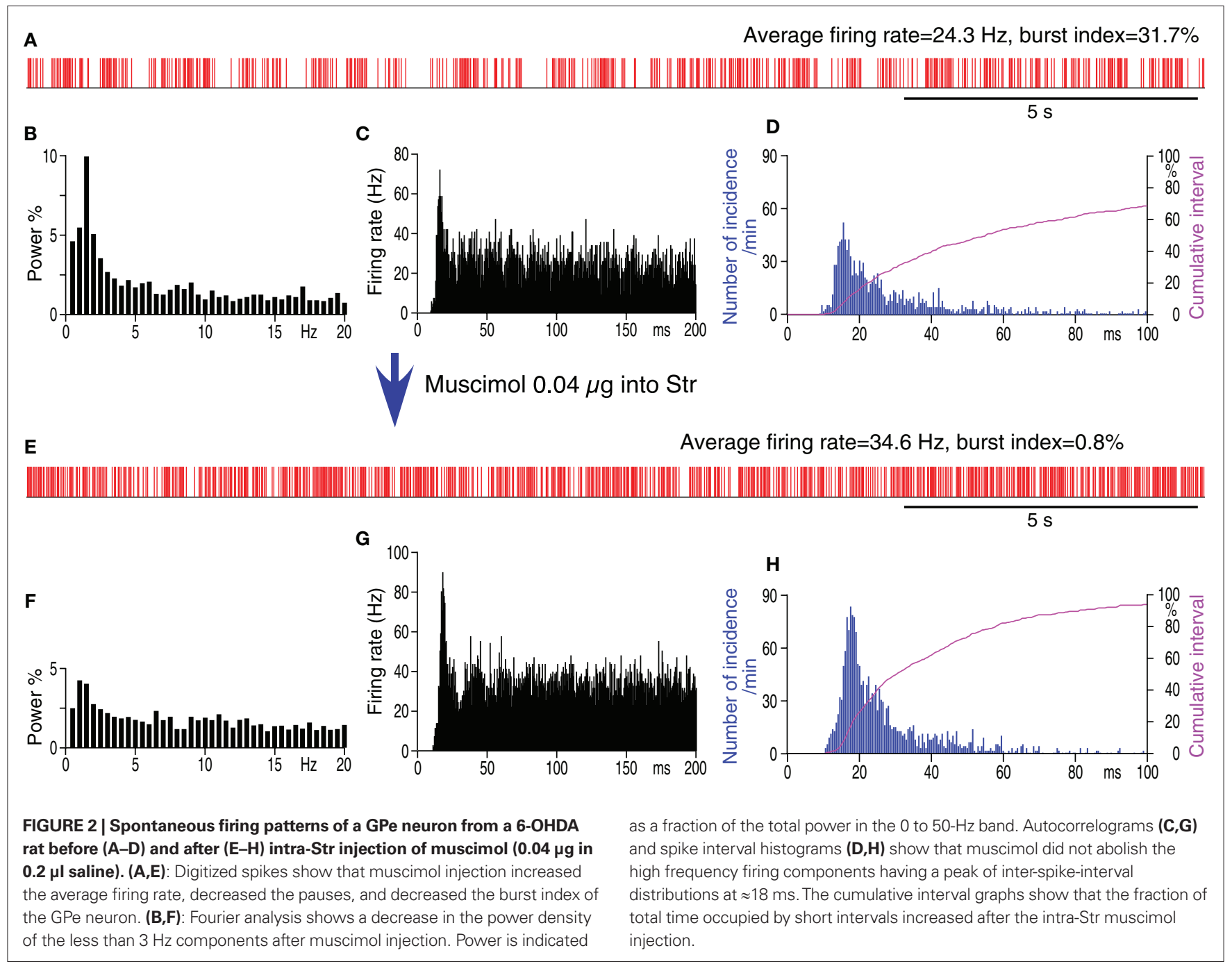

\section{FIRING PATTERN OF Str NEURONS}

Striatal projection neurons in anesthetized normal rats show spontaneous membrane potential shifts between hyperpolarized and depolarized states, and spike firings occur in groups during the depolarized states (Wilson and Groves, 1981). Thus, the firing pattern of Str projection neurons differs from both the tonic firing with large single or doublet spikes of putative cholinergic interneurons and the high frequency firing with sharp spikes of putative parvalbumin-containing interneurons. Intracellular recordings in halothane or urethane-anesthetized rats reported that DA-depletion significantly increased the number of spikes during the depolarized states (Pang et al., 2001; Tseng et al., 2001). These previous findings together with the results of the present intra-Str muscimol injection experiments suggest that Str-GPe inputs increase the pauses and bursts in firings of GPe neurons. To study changes in the activity pattern of Str neurons after DA-depletion in more detail, the spontaneous activity of possible projection neurons was recorded. 
Under the present experimental conditions, spontaneously active putative projection neurons were only occasionally found in the Str of control rats, although MC stimulation disclosed numerous silent or only occasionally firing neurons when these neurons responded with an excitation to the stimulation. We recorded 11 spontaneously active neurons with an average firing rate of $0.5-3.8 \mathrm{~Hz}$ in the sensorimotor territory of the Str of control rats (Figure 3A; Table 3). Figure 3A shows that burst firings of Str neurons tend to occur in groups with an inter burst interval of approximately $1 \mathrm{~Hz}$, as seen in the frequency distribution histogram (Figure 3B). The spontaneously active putative projection neurons were more frequently encountered in the Str of 6-OHDA than in normal rats. In 6-OHDA rats, 22 neurons with irregular spontaneous activity of 1.1-11.6 Hz were recorded from the sensorimotor territory of the Str (Figure 3D; Table 3). The frequency distribution histograms (Figures 3B,E) and Table 3 show that burst firings occur more frequently with shorter inter burst intervals in the Str of 6-OHDA than in control rats. The burst index was slightly but significantly lower in the neurons from 6-OHDA rats. Figures 3C,F show population inter-spike-interval distributions calculated from all recorded neurons. Both histograms show that short range, $>100 \mathrm{~ms}$, inter-spikeinterval distributions are similar and have a peak at about $10 \mathrm{~ms}$.

Table 4 compares the characteristics of the 121 and 442 bursts detected in the 11 neurons from control and the 22 neurons from 6-OHDA rats, respectively. The duration and the surprise value of bursts were slightly but significantly decreased in the 6-OHDA group. However, the number of spikes per burst increased in this group. These observations are consistent with the report that DA-depletion made up- and down-state transitions more distinct and that a larger proportion of up-states, even short ones, triggered spikes (Tseng et al., 2001). Eight of the 22 Str neurons from 6-OHDA rats were juxtacellularly labeled with Neurobiotin. They were all medium spiny neurons, and the main axons of five of the neurons were determined to have terminal fields confined to GPe (Figure 4), but the other three faded out before entering GPe. These data suggest that DA-depletion increased the spontaneous activity of Str-GPe projection neurons, and the increase is accompanied with an increased occurrence of stronger burst firings.

Table 3 | Properties of spontaneous firings of Str neurons in control and 6-OHDA rats.

\begin{tabular}{llll}
\hline & Normal, $\boldsymbol{n = 1 1}$ & & 6-OHDA, $\boldsymbol{n = 2 2}$ \\
\hline Firing rate $(\mathrm{Hz})$ & $2.1 \pm 1.2$ & $<0.0001$ & $6.4 \pm 2.7$ \\
Burst index $(\%)$ & $73.5 \pm 9.1$ & $<0.02$ & $61.8 \pm 13.1$ \\
Burst interval (s) & $4.6 \pm 2.6$ & $<0.01$ & $2.4 \pm 1.0$ \\
\hline
\end{tabular}

Burst index: fraction of spikes in bursts over a total number of spikes. Data: mean \pm SD; Statistics: Mann-Whitney U-test.

Table 4 | Properties of bursts of Str neurons in control and 6-OHDA rats.

\begin{tabular}{llll}
\hline & Normal, $\boldsymbol{n = 1 2 1}$ & & 6-OHDA, $\boldsymbol{n = 4 4 2}$ \\
\hline Burst duration (ms) & $340 \pm 339$ & $<0.02$ & $264 \pm 334$ \\
Surprise value & $9.2 \pm 5.3$ & $<0.05$ & $8.5 \pm 8.5$ \\
Number of spikes/burst & $6.9 \pm 4.2$ & $<0.0001$ & $9.0 \pm 8.6$ \\
\hline
\end{tabular}

Data: mean \pm SD; statistics: Mann-Whitney U-test.

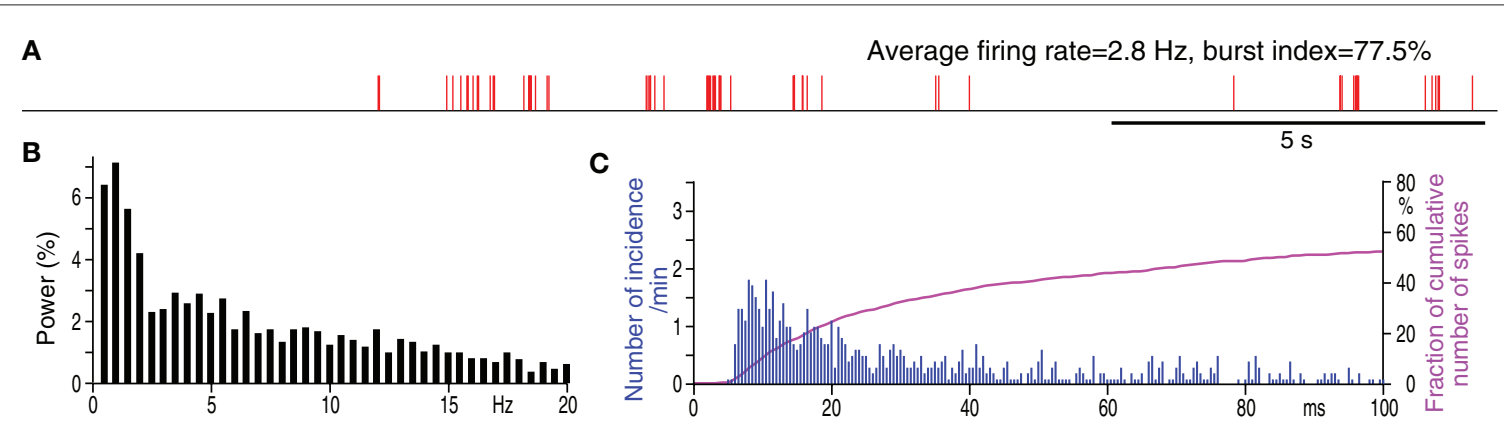

D

Average firing rate $=10.1 \mathrm{~Hz}$, burst index $=56.0 \%$
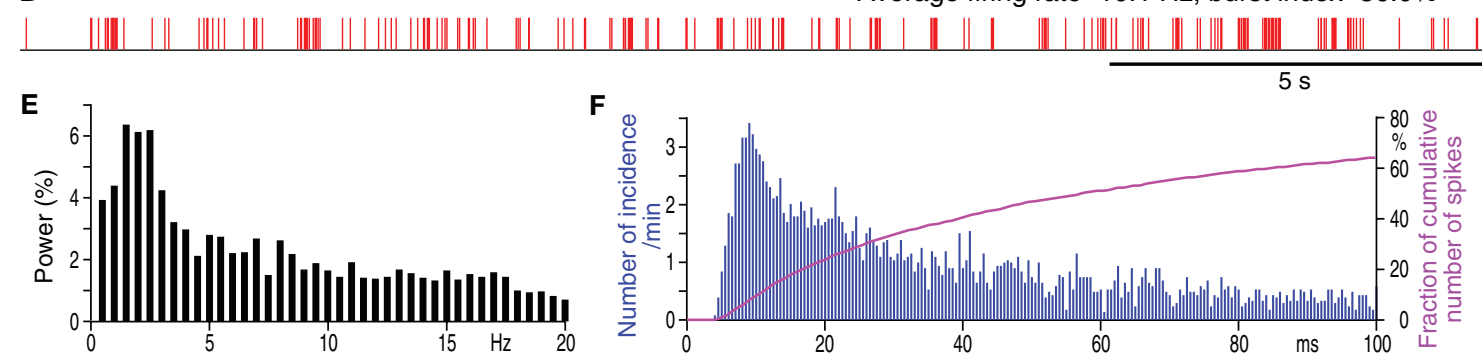

FIGURE 3 | Spontaneous firing patterns of a Str neuron from a control (A-C) and from a 6-OHDA rat (D-F). (A,D): digitized spikes show groups of firings with very high burst indices in both neurons from the control and from the 6-OHDA Str. Spike interval histograms (C,F) show that the neurons from both rats have similar shorter inter-spike-interval distributions. 

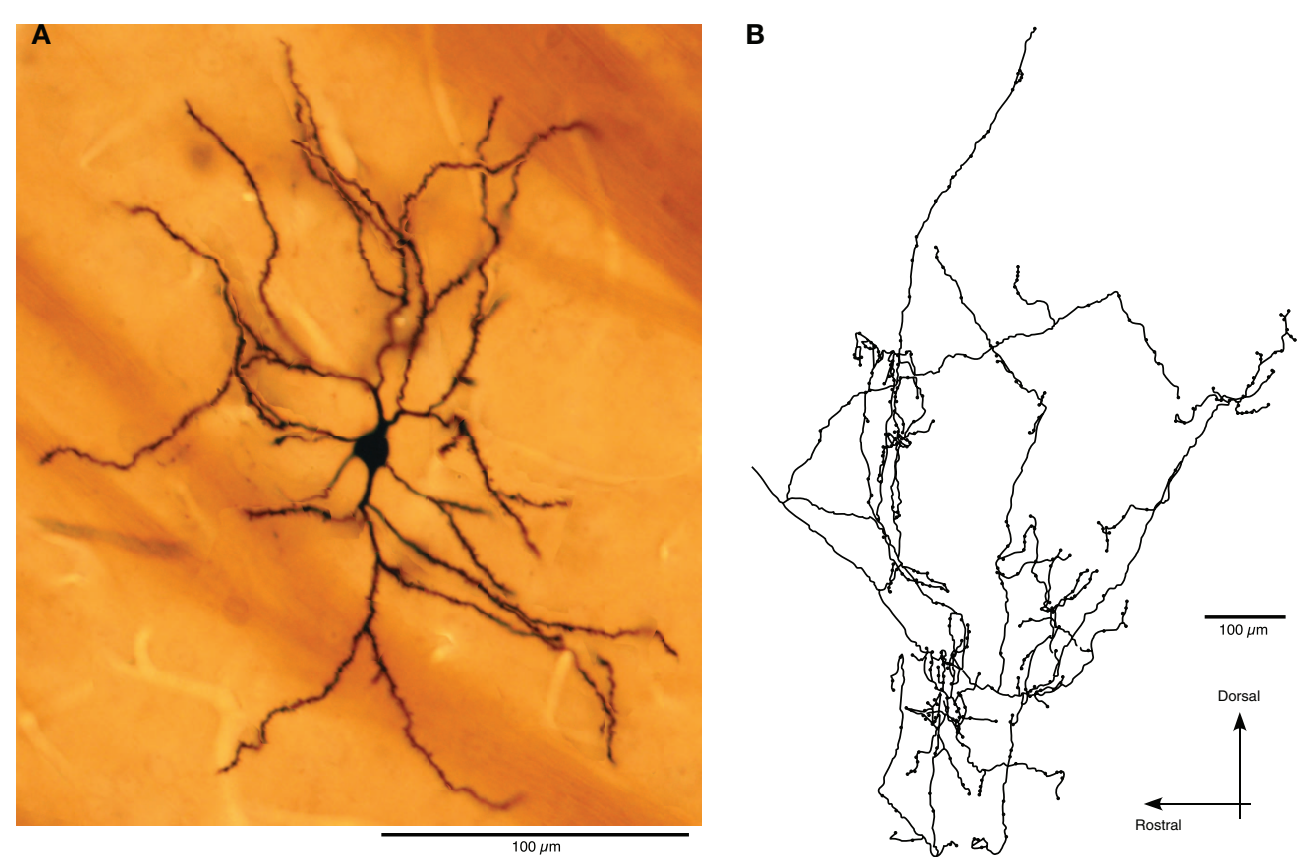

FIGURE 4 |An example of juxtacellulary stained spontaneously active Str neurons. (A): Photomontage shows a medium spiny neuron. (B): A camera lucida drawing of the axonal arbor of the neuron in GPe.

\section{METHYL-L-DOPA INJECTION INTO Str}

To evaluate to what degree the increased pauses and bursts of GPe neurons could be attributed to DA-depletion in Str, the acute effects of intra-Str injection of the DA precursor methyl-L-DOPA on the firing of GPe neurons in 6-OHDA rats were examined. The tip of the methyl-L-DOPA injection pipette was placed in the sensorimotor territory of the Str, in a similar manner to the muscimol injection described above. The neurons in the sensorimotor territory of GPe were recorded before and 10-20 min after the methyl-L-DOPA injections ( $4 \mu \mathrm{g}$ in $0.2 \mu \mathrm{l}$ saline, $0.04 \mu \mathrm{l} / \mathrm{min}$ ). This dose of methylL-DOPA was based on behavioral tests that determined the amount that changed the preferred turning direction from left to right and also increased the total distance moved during the test period (Kita and Kita, 2011). Figure 5 and Table 2 show that methyl-L-DOPA significantly increased the average firing rate and decreased the fraction of spikes in bursts and the fraction of time occupied by pauses. Methyl-L-DOPA also decreased the $<3 \mathrm{~Hz}$ components of the frequency distribution. The autocorrelograms and inter-spikeinterval distributions show that methyl-L-DOPA shifted the peak of the inter-spike-interval distribution to the left and did not abolish the high frequency component of firings with short inter-spike-intervals. These changes were very similar to those resulting from intra-Str muscimol injections. These results indicate that acute replacement of DA in Str can reduce burst and pause generation in GPe.

\section{DISCUSSION}

\section{RECORDING CONDITIONS}

The present unit recordings were performed under $0.5-1 \%$ isoflurane anesthesia. We believe that the anesthetized rats offer more homogenous conditions for unit activity recording than awake rats because monitoring the movements and finding quietly resting periods that are long enough for data acquisition is very difficult with awake rats. For instance, even after acclimating the awake rats to $2-3 \mathrm{~h}$ of restraint, they often move their whiskers; this whisking related unit activity, probably including both movement initiation and movement evoked sensory afferent inputs, could be often found in Str and GPe (Kita, unpublished observation). During unit recordings, taking advantage of the quick adjustment capability of gas inhalation anesthesia, the anesthesia level was adjusted to eliminate spontaneous whisker movements as well as any other body movement to MC stimulation. Under these conditions, the rats did not show movement responses to light touch but responded to painful stimuli such as a foot pinch. The level of anesthesia judged by electrocorticogram observation was stage two or three sleep but not slow wave sleep (data not shown). These levels were chosen to avoid the induction of strong, synchronized, slow oscillations during slow wave sleep that is induced by higher isoflurane doses. Under slow wave sleep, cortical, Str neurons, and probably thalamic neurons undergo synchronized, slow oscillation, also known as up- and down-state or depolarized-and hyperpolarized-state (Stern et al., 1997). Probably because of this wide cortical synchrony, many Str projection neurons fire during the up-state. It has also been shown that the slow oscillation changes after DA-depletion (Pang et al., 2001; Mallet et al., 2006). However, Str projection neurons are almost silent in normal quietly resting awake rats and monkeys. Our recording conditions were set to create the Str activity resembling that of quietly resting awake animals. The present data show that a significant increase in burst firing can be observed in GPe of 6-OHDA rats anesthetized with isoflurane. 


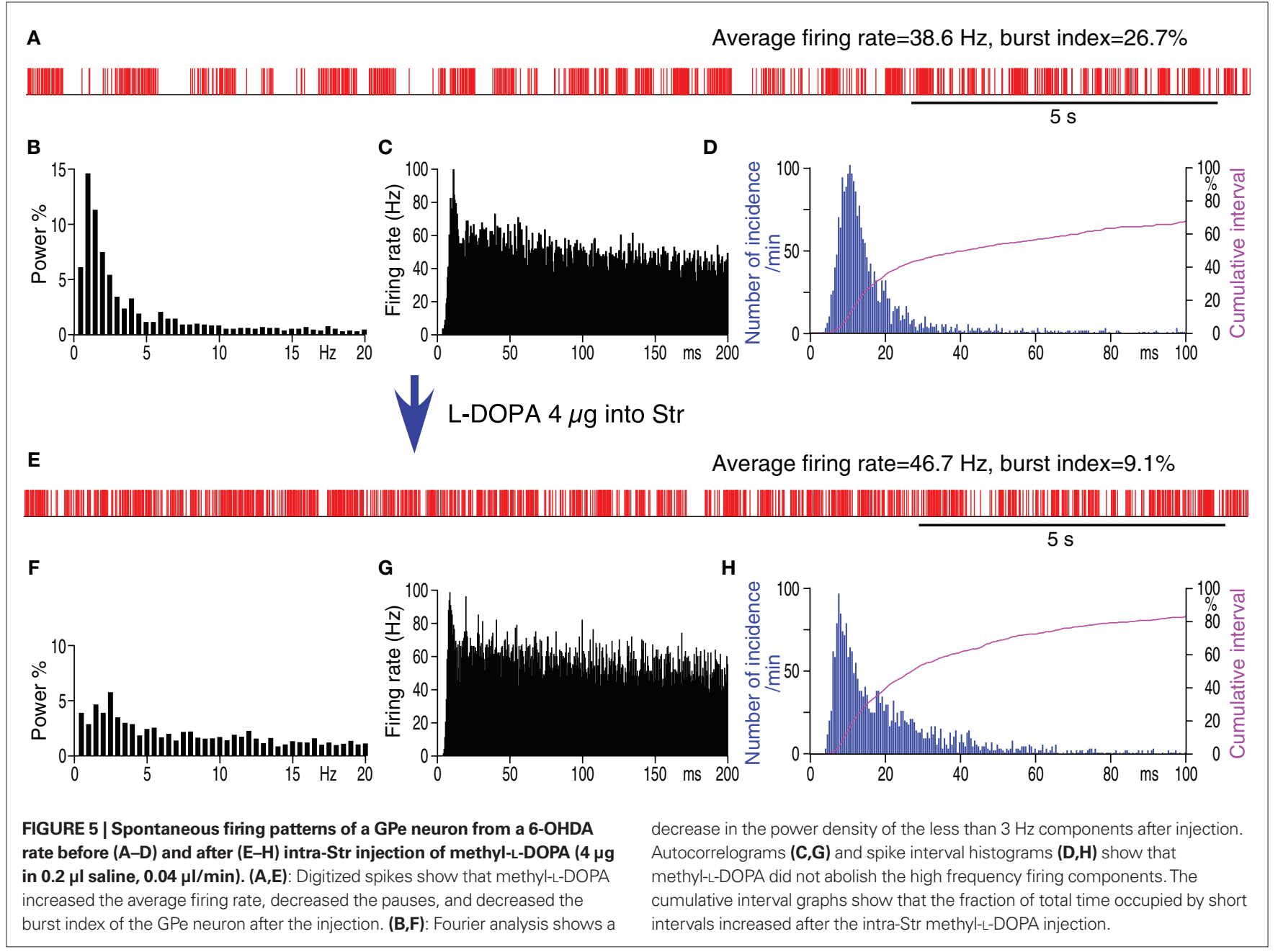

\section{INCREASED Str INPUTS SIGNIFICANTLY INCREASE PAUSES AND BURSTS OF GPe NEURONS AFTER DA-DEPLETION}

The most common firing type of GPe neurons is high frequency firing with pauses (DeLong, 1971). The pauses are most likely triggered by GABAergic inputs based on the following reported observations: a local injection of the $\mathrm{GABA}_{\mathrm{A}}$ antagonist gabazine eliminates most of pauses (Kita et al., 2004), a burst activation of Str inputs can induce a long inhibition in GPe (Kaneda and Kita, 2005), burst firings of Str neurons are synchronous with pauses of GPe firings (Walters et al., 2007), and pauses are not preceded by bursts rejecting involvement of calcium-activated potassium current (Elias et al., 2007). Also, a number of studies have shown an increased activity of Str neurons after DA-depletion (Pang et al., 2001; Tseng et al., 2001). Based on these data, we formed the hypothesis that DA-depletion increases burst firings of Str neurons projecting to the GPe, which then generate pauses in the firing of GPe neurons, which then finally contribute to the generation of burst firing in the GPe itself and in the projection sites of GPe. This study provides key supportive data for the hypothesis that silencing Str by local injection of muscimol significantly decreased pauses and bursts of GPe firings.
The membrane potential of Str projection neurons in normal rats is periodically shifted between hyperpolarized and depolarized states by cortical inputs, with spike firings occurring only during the depolarized states (Wilson and Groves, 1981; Stern et al., 1998). In control rats, the depolarized states are mostly subthreshold, with only occasional firings during these states. After DA-depletion, the depolarized states reach the threshold more frequently due to altered glutamatergic and GABAergic inputs and altered membrane properties of the neurons; thus firing increases during DA-depleted depolarized states (Chen et al., 2001; Pang et al., 2001; Tseng et al., 2001; West and Grace, 2002; Mallet et al., 2006). The present data are consistent with the previous observations that the occurrence of bursts in Str neurons and the average number of spikes per burst significantly increased after DA-depletion. Juxtacellular labeling results suggest that most of the burst firing of Str neurons are from those that project only to GPe. The present data also revealed two new aspects: One is that bursts tend to occur in groups in both normal and DA-depleted Str, suggesting a very low, $\approx 0.1 \mathrm{~Hz}$, oscillation that can be seen in the both conditions. Similar, very slow oscillations were reported in other nuclei of basal ganglia and other brain areas (Walters and Bergstrom, 2010). The second is that distributions of short inter-spike-intervals are similar in control and DA-depleted Str, suggesting that bursts 
with short inter-spike-intervals occur in normal Str. Str-GPe synapses should be significantly augmented during the burst activation and evoke strong $\mathrm{GABA}_{\mathrm{A}}$ and $\mathrm{GABA}_{\mathrm{B}}$ receptor mediated IPSPs (Kaneda and Kita, 2005; Sims et al., 2008) and alter opioid receptor mediated modulations of ionic currents in GPe neurons (Ogura and Kita, 2000; Spadoni et al., 2004). The Str-GPe inhibition may also be increased by removal of pre- and postsynaptic DA suppression of Str-GPe synapses (Floran et al., 1997; Cooper and Stanford, 2001; Shin et al., 2003; Watanabe et al., 2009). Thus, Str burst firings will effectively inhibit GPe neurons. However, it has to be noted that these observations do not provide strong evidence that the GABAergic Str burst inputs always directly cause the hyperpolarizations for pauses. It has been documented that GPe neurons in slice preparations generated pauses when they were hyperpolarized by a continuous somatic current injection (Hashimoto and Kita, 2006; Deister et al., 2009). The increased GABAergic inputs from Str and the increased glutamatergic inputs from STN can create a condition such that timely arrival of GABAergic or glutamatergic inputs can flip the membrane of GPe neurons between hyperpolarized and depolarized states (Hashimoto and Kita, 2006). These pauses could contribute to the increase in burst activity of GPe neurons commonly observed in patients and animal models of PD. Pauses can intensify burst firings of GPe itself by the rebound excitation mechanism (i.e., deinactivation of Na-channels during hyperpolarization) and by the GPe-STN-GPe negative feedback inputs. Our data suggest that the pauses in GPe firing can generate strong disinhibition bursts in the STN and GPi in 6-OHDA rats (Kita, unpublished observation), though this may not be the significant factor in normal animals (Elias et al., 2008).

\section{CONTRIBUTION OF STN INPUTS IN THE BURST GENERATION IN GPe}

The GPe receives a major excitatory input from STN, and the burst activity and mean firing rate of STN increase significantly after DA-depletion (Magill et al., 2001; Bevan et al., 2002; Sharott et al., 2005; Galvan and Wichmann, 2008; Israel and Bergman, 2008; Galati et al., 2009). It has also been shown in urethane-anesthetized rats that acute DA-depletion by chemical blockade of the medial forebrain bundle significantly increased acute pathological synchronization of the cortico-STN-GPe pathway (Galati et al., 2009). The increased burst activity in STN may be in part due to the increased burst activity of GPe as well as to the increased cortico-STN synchronous inputs, changes in pedunculopontine inputs, and altered membrane properties of STN neurons (Shen and Johnson, 2000, 2005; Baufreton and Bevan, 2008; Dejean et al., 2008; Galati et al., 2009). This study shows that intra-Str injection of muscimol in 6-OHDA rats did not abolish a high frequency (inter-spike-interval peak $\approx 18 \mathrm{~ms}$ ) component of firing, though it significantly decreased bursts, pauses, and the $<3 \mathrm{~Hz}$ components of the firing frequency distribution of the firings in GPe neurons. A study in normal monkey GPe showed that a combined local injection of $\mathrm{GABA}_{\mathrm{A}}$ and glutamate antagonists is required to regularize firings of GPe neurons (Kita et al., 2004). Thus, both Str and STN inputs contribute to the generation of burst activity in GPe.

\section{REFERENCES}

Albin, R. L., Young, A. B., and Penney, J. B. (1989). The functional anatomy of basal ganglia disorders. Trends Neurosci. 12, 366-375.
Bamford, N. S., Zhang, H., Schmitz, Y., Wu, N. P., Cepeda, C., Levine, M. S., Schmauss, C.,Zakharenko,S.S.,Zablow, L., and Sulzer,D. (2004). Heterosynaptic dopamine neurotransmission selects

\section{AVERAGE FIRING RATE OF GPe NEURONS}

Studies in animal models of PD reported either no change or a significant decrease in the average firing rate of GPe neurons (Filion, 1979; Pan and Walters, 1988; Albin et al., 1989; DeLong, 1990; Filion and Tremblay, 1991; Ni et al., 2000; Magill et al., 2001; Soares et al., 2004; Mallet et al., 2008). In the present recording conditions, the average firing rate of GPe neurons did not decrease significantly in spite of the increased Str inhibition. It has also been shown that DA-depletion down regulates HCN2 channel expressions in GPe neurons, though it normally enhances autonomous firing of neurons (Chan et al., 2011). This unchanged average firing rate suggests that these increased inhibitory forces were counter-balanced by increased excitatory inputs from STN, because a significant increase in the firing rate of STN neurons after DA-depletion is a commonly reported observation (Hollerman and Grace, 1992; Bergman et al., 1994; Magill et al., 2001; Pelled et al., 2002; Wichmann and DeLong, 2006).

\section{EFFECTS OF INTRA-Str METHYL-L-DOPA INJECTION}

Injection of a behaviorally effective dose of methyl-L-DOPA into the Str of 6-OHDA rats decreased the pauses and bursts of GPe neurons. These changes were attributed to the decreased spontaneous burst activity of Str-GPe neurons. DA-depletion increases D2-R expression in Str-GPe neurons (Gerfen et al., 1990) and also sensitizes D2-Rs on cortico-Str terminals (Bamford et al., 2004). Thus, the changes may be due to activation of these D2-Rs by DA converted from L-DOPA in Str (Xu and Dluzen, 1996; Tanaka et al., 1999). The elucidation of the L-DOPA effects on spontaneous firing and the cortical and thalamic stimulation-induced responses of Str neurons will be the subjects of future investigations.

\section{FUNCTIONAL IMPLICATIONS}

This report documents that DA-depletion increases the spontaneous activity of some Str-GPe projection neurons and that the increased Str-GPe inputs play significant roles in the generation of pauses and bursts of firings in GPe neurons. This study also shows that intra-Str injection of a behaviorally effective dose of methyl-L-DOPA reduces these pauses and bursts. We infer from these observations that DA-depleted Str generates abnormal signals in GPe that can be transmitted to the basal ganglia output nuclei $\mathrm{GPi} / \mathrm{SNr}$, which then disables information processing capability at their projection sites in the thalamus and brainstem that may lead to the manifestation of psychomotor disabilities in PD (Stefani et al., 2011). It can be further suggested that treatment to reduce burst Str-GPe inhibitory inputs may help to restore some of these disabilities.

\section{ACKNOWLEDGMENTS}

This work was supported by the National Institute of Neurological Disorders and Stroke Grants NS-47085 and NS-57236. We thank R. Kita for editing the manuscript and J. Butler for technical assistance.

sets of corticostriatal terminals. Neuron 42, 653-663.

Baufreton, J., and Bevan, M. D. (2008) D2-like dopamine receptor-mediated modulation of activity-dependent plasticity at GABAergic synapses in the subthalamic nucleus. J. Physiol. (Lond.) 586, 2121-2142.

Bergman, H., Wichmann, T., Karmon, B., and DeLong, M.R.(1994). The primate 
subthalamic nucleus. II. Neuronal activity in the MPTP model of parkinsonism. J. Neurophysiol. 72, 507-520.

Bevan, M. D., Magill, P. J., Terman, D., Bolam, J. P., and Wilson, C. J. (2002). Move to the rhythm: oscillations in the subthalamic nucleus-external globus pallidus network. Trends Neurosci. 25, 525-531.

Chan, C. S., Glajch, K. E., Gertler, T. S., Guzman, J. N., Mercer, J. N., Lewis, A. S., Goldberg, A. B., Tkatch, T., Shigemoto, R., Fleming, S. M., Chetkovich, D. M., Osten, P., Kita, H., and Surmeier, D. J. (2011). HCN channelopathy in external globus pallidus neurons in models of Parkinson's disease. Nat. Neurosci. 14, 85-92.

Chen, M. T., Morales, M., Woodward, D. J., Hoffer, B. J., and Janak, P. H. (2001). In vivo extracellular recording of striatal neurons in the awake rat following unilateral 6-hydroxydopamine lesions. Exp. Neurol. 171, 72-83.

Cooper, A. J., and Stanford, I. M. (2001). Dopamine D2 receptor mediated presynaptic inhibition of striatopallidal GABA(A) IPSCs in vitro. Neuropharmacology 41, 62-71.

Deister, C. A., Chan, C. S., Surmeier, D. J., and Wilson, C. J. (2009). Calciumactivated SK channels influence voltage-gated ion channels to determine the precision of firing in globus pallidus neurons. J. Neurosci. 29, 8452-8461.

Dejean, C., Gross, C. E., Bioulac, B., and Boraud, T. (2008). Dynamic changes in the cortex-basal ganglia network after dopamine depletion in the rat. J. Neurophysiol. 100, 385-396.

DeLong, M. R. (1971). Activity of pallidal neurons during movement. $J$. Neurophysiol. 34, 414-427.

DeLong, M. R. (1990). Primate models of movement disorders of basal ganglia origin. Trends Neurosci. 13, 281-285.

Elias, S., Joshua, M., Goldberg, J. A., Heimer, G., Arkadir, D., Morris, G., and Bergman, H. (2007). Statistical properties of pauses of the high-frequency discharge neurons in the external segment of the globus pallidus. $J$. Neurosci. 27, 2525-2538.

Elias, S., Ritov, Y., and Bergman, H. (2008). Balance of increases and decreases in firing rate of the spontaneous activity of basal ganglia high-frequency discharge neurons. J. Neurophysiol. 100, 3086-3104.

Filion, M. (1979). Effects of interruption of the nigrostriatal pathway and of dopaminergic agents on the spontaneous activity of globus pallidus neurons in the awake monkey. Brain Res. 178, 425-441.

Filion, M., and Tremblay, L. (1991). Abnormal spontaneous activity of globus pallidus neurons in monkeys with MPTP-induced parkinsonism. Brain Res. 547, 142-151.

Floran, B., Floran, L., Sierra, A., and Aceves, J. (1997). D2 receptor-mediated inhibition of GABA release by endogenous dopamine in the rat globus pallidus. Neurosci. Lett. 237, $1-4$.

Flores-Barrera, E., Vizcarra-Chacon, B. J., Tapia, D., Bargas, J., and Galarraga, E. (2010). Different corticostriatal integration in spiny projection neurons from direct and indirect pathways. Front. Syst. Neurosci. 4:15. doi: 10.3389/fnsys.2010.00015

Galati, S., Stanzione, P., D’Angelo, V., Fedele, E., Marzetti, F., Sancesario, G., Procopio, T., and Stefani, A. (2009). The pharmacological blockade of medial forebrain bundle induces an acute pathological synchronization of the cortico-subthalamic nucleusglobus pallidus pathway. J. Physiol. (Lond.) 587, 4405-4423.

Galvan, A., and Wichmann, T. (2008). Pathophysiology of parkinsonism. Clin. Neurophysiol. 119, 1459-1474.

Gerfen, C. R., Engber, T. M., Mahan, L. C., Susel, Z., Chase, T. N., Monsma, F. J. Jr., and Sibley, D. R. (1990). D1 and $\mathrm{D} 2$ dopamine receptor-regulated gene expression of striatonigral and striatopallidal neurons. Science 250, 1429-1432.

Hashimoto, K., and Kita, H. (2006). Slow oscillatory activity of rat globus pallidus neurons in vitro. Eur. J. Neurosci. $23,443-453$.

Hernandez-Lopez, S., Tkatch, T., PerezGarci, E., Galarraga, E., Bargas, J., Hamm, H., and Surmeier, D. J. (2000). D2 dopamine receptors in striatal medium spiny neurons reduce L-type $\mathrm{Ca} 2+$ currents and excitability via a novel PLC[beta]1-IP3-calcineurinsignaling cascade. J. Neurosci. 20, 8987-8995.

Hollerman, J. R., and Grace, A. A. (1992). Subthalamic nucleus cell firing in the 6-OHDA-treated rat: basal activity and response to haloperidol. Brain Res. 590, 291-299.

Israel, Z., and Bergman, H. (2008). Pathophysiology of the basal ganglia and movement disorders: from animal models to human clinical applications. Neurosci. Biobehav. Rev. 32, 367-377.

Kaneda, K., and Kita, H. (2005). Synaptically released GABA activates both pre- and postsynaptic GABA(B) receptors in the rat globus pallidus. $J$. Neurophysiol. 94, 1104-1114.

Kita,H., and Kita, T. (2011). Cortical stimulation evokes abnormal responses in the dopamine-depleted rat basal ganglia. J. Neurosci. in press.

Kita, H., Nambu, A., Kaneda, K., Tachibana, Y., and Takada, M. (2004).
Role of ionotropic glutamatergic and GABAergic inputs on the firing activity of neurons in the external pallidum in awake monkeys. J. Neurophysiol. 92 , 3069-3084.

Legendy, C. R., and Salcman, M. (1985), Bursts and recurrences of bursts in the spike trains of spontaneously active striate cortex neurons. J. Neurophysiol. 53, 926-939.

Magill, P. J., Bolam, J. P., and Bevan, M. D. (2001). Dopamine regulates the impact of the cerebral cortex on the subthalamic nucleus-globus pallidus network. Neuroscience 106, 313-330.

Mallet, N., Ballion, B., Le Moine, C., and Gonon, F. (2006). Cortical inputs and GABA interneurons imbalance projection neurons in the striatum of parkinsonian rats. J. Neurosci. 26, 3875-3884.

Mallet, N., Pogosyan, A., Marton, L. F., Bolam, J. P., Brown, P., and Magill, P. J. (2008). Parkinsonian beta oscillations in the external globus pallidus and their relationship with subthalamic nucleus activity. J. Neurosci. 28, 14245-14258.

Ni, Z., Bouali-Benazzouz, R., Gao, D. Benabid, A. L., and Benazzouz, A. (2000). Changes in the firing pattern of globus pallidus neurons after the degeneration of nigrostriatal pathway are mediated by the subthalamic nucleus in the rat. Eur. J. Neurosci. 12 , 4338-4344.

Ogura, M., and Kita, H. (2000). Dynorphin exerts both postsynaptic and presynaptic effects in the globus pallidus of the rat. J. Neurophysiol. 83, 3366-3376

Pan, H. S., and Walters, J. R. (1988). Unilateral lesion of the nigrostriatal pathway decreases the firing rate and alters the firing pattern of globus pallidus neurons in the rat. Synapse 2 , 650-656.

Pang, Z., Ling, G. Y., Gajendiran, M., and Xu, Z.C. (2001). Enhanced excitatory synaptic transmission in spiny neurons of rat striatum after unilateral dopamine denervation. Neurosci. Lett. 308, 201-205.

Pelled, G., Bergman, H., and Goelman, G. (2002). Bilateral overactivation of the sensorimotor cortex in the unilateral rodent model of Parkinson's disease -a functional magnetic resonance imaging study. Eur. J. Neurosci. 15,389-394.

Pinault, D. (1996). A novel single-cell staining procedure performed in vivo under electrophysiological control: morpho-functional features of juxtacellularly labeled thalamic cells and other central neurons with biocytin or neurobiotin. J. Neurosci. Methods 65, 113-136.

Sharott, A., Magill, P. J., Harnack, D., Kupsch, A., Meissner, W., and Brown,
P. (2005). Dopamine depletion increases the power and coherence of beta-oscillations in the cerebral cortex and subthalamic nucleus of the awake rat. Eur. J. Neurosci. 21, 1413-1422.

Shen, K. Z., and Johnson, S. W. (2000). Presynaptic dopamine D2 and muscarine $\mathrm{M} 3$ receptors inhibit excitatory and inhibitory transmission to rat subthalamic neurones in vitro. J. Physiol. (Lond.) 525(Pt 2), 331-341.

Shen, K. Z., and Johnson, S. W. (2005). Dopamine depletion alters responses to glutamate and GABA in the rat subthalamic nucleus. Neuroreport 16 , 171-174.

Shen, W., Flajolet, M., Greengard, P., and Surmeier, D. J. (2008). Dichotomous dopaminergic control of striatal synaptic plasticity. Science 321, 848-851.

Shin, R. M., Masuda, M., Miura, M., Sano, H., Shirasawa, T., Song, W. J., Kobayashi, K., and Aosaki, T. (2003). Dopamine D4 receptor-induced postsynaptic inhibition of GABAergic currents in mouse globus pallidus neurons. J. Neurosci. 23, 11662-11672.

Sims, R. E., Woodhall, G. L., Wilson, C. L. and Stanford, I. M. (2008). Functional characterization of GABAergic pallidopallidal and striatopallidal synapses in the rat globus pallidus in vitro. Eur. J. Neurosci. 28, 2401-2408.

Soares, J., Kliem, M. A., Betarbet, R., Greenamyre, J. T., Yamamoto, B., and Wichmann, T. (2004). Role of external pallidal segment in primate parkinsonism: comparison of the effects of 1-methyl-4-phenyl-1,2,3,6-tetrahydropyridine-induced parkinsonism and lesions of the external pallidal segment. J. Neurosci. 24, 6417-6426.

Spadoni, F., Martella, G., Martorana, A., Lavaroni, F., D'Angelo, V., Bernardi, G., and Stefani, A. (2004). Opioidmediated modulation of calcium currents in striatal and pallidal neurons following reserpine treatment: focus on kappa response. Synapse 51 194-205.

Stefani, A., Fedele, E., Pierantozzi, M., Galati, S., Marzetti, F., Peppe, A., Pastore, F. S., Bernardi, G., and Stanzione, P. (2011). Reduced GABA content in the motor thalamus during effective deep brain stimulation of the subthalamic nucleus. Front. Syst. Neurosci. 5:17. doi: 10.3389/ fnsys.2011.00017

Stefani, A., Spadoni, F., Martorana, A., Lavaroni, F., Martella, G., Sancesario, G., and Bernardi, G. (2002). D2-mediated modulation of N-type calcium currents in rat globus pallidus neurons following dopamine denervation. Eur. J. Neurosci. 15, 815-825.

Stern, E. A., Jaeger, D., and Wilson, C. J. (1998). Membrane potential syn- 
chrony of simultaneously recorded striatal spiny neurons in vivo. Nature 394, 475-478.

Stern, E. A., Kincaid, A. E., and Wilson, C. J. (1997). Spontaneous subthreshold membrane potential fluctuations and action potential variability of rat corticostriatal and striatal neurons in vivo. J. Neurophysiol. 77, 1697-1715.

Surmeier, D. J., Shen, W., Day, M., Gertler, T., Chan, S., Tian, X., and Plotkin, J. L. (2010). The role of dopamine in modulating the structure and function of striatal circuits. Prog. Brain Res. 183, 149-167.

Tanaka, H., Kannari, K., Maeda, T., Tomiyama, M., Suda, T., and Matsunaga, M. (1999). Role of serotonergic neurons in L-DOPA-derived extracellular dopamine in the striatum of 6-OHDA-lesioned rats. Neuroreport 10, 631-634.

Tseng, K. Y., Kasanetz, F., Kargieman, L., Riquelme, L. A., and Murer, M.
G. (2001). Cortical slow oscillatory activity is reflected in the membrane potential and spike trains of striatal neurons in rats with chronic nigrostriatal lesions. J. Neurosci. 21, 6430-6439.

Walters, J. R., and Bergstrom, D. A. (2010). "Synchronous activity in basal ganglia circuits," in Handbook of Basal Ganglia Structure and Function, eds S. Heinz Steiner and K. Y. Tseng (Philadelphia, PA: Elsevier), 429-443.

Walters, J. R., Hu, D., Itoga, C. A., ParrBrownlie, L. C., and Bergstrom, D. A. (2007). Phase relationships support a role for coordinated activity in the indirect pathway in organizing slow oscillations in basal ganglia output after loss of dopamine. Neuroscience 144, 762-776.

Watanabe, K., Kita, T., and Kita, H. (2009). Presynaptic actions of $\mathrm{d} 2$-like receptors in the rat cortico-striato-globus pallidus disynaptic connection in vitro. J. Neurophysiol. 101, 665-671.
West, A. R., and Grace, A. A. (2002). Opposite influences of endogenous dopamine D1 and D2 receptor activation on activity states and electrophysiological properties of striatal neurons: studies combining in vivo intracellular recordings and reverse microdialysis. J. Neurosci. 22, 294-304.

Wichmann, T., and DeLong, M. R. (2006). Basal ganglia discharge abnormalities in Parkinson's disease. J. Neural Transm. Suppl. 21-25.

Wichmann, T., and Soares, J. (2006). Neuronal firing before and after burst discharges in the monkey basal ganglia is predictably patterned in the normal state and altered in parkinsonism. $J$. Neurophysiol. 95, 2120-2133.

Wilson, C. J., and Groves, P. M. (1981). Spontaneous firing patterns of identified spiny neurons in the rat neostriatum. Brain Res. 220, 67-80.

Xu, K., and Dluzen, D. E. (1996). L-DOPA modulation of corpus striatal dopamine and dihydroxyphenylacetic acid output from intact and 6-OHDA lesioned rats. J. Neural Transm. 103, 1295-1305.

Conflict of Interest Statement:The authors declare that the research was conducted in the absence of any commercial or financial relationships that could be construed as a potential conflict of interest.

Received: 07 April 2011; paper pending published: 03 May 2011; accepted: 27 May 2011; published online: 08 June 2011.

Citation: Kita H and Kita T (2011) Role of striatum in the pause and burst generation in the globus pallidus of 6-OHDAtreated rats. Front. Syst. Neurosci. 5:42. doi: 10.3389/fnsys.2011.00042

Copyright (C) 2011 Kita and Kita. This is an open-access article subject to a nonexclusive license between the authors and Frontiers Media SA, which permits use, distribution and reproduction in other forums, provided the original authors and source are credited and other Frontiers conditions are complied with. 